\title{
Diagnosis and management of childhood sleep-disordered breathing
}

\author{
Clinical approach
}

Abdullah Al-Shamrani, MD, FRCPCH, Adel S. Alharbi, MD, FCCP.

\begin{abstract}

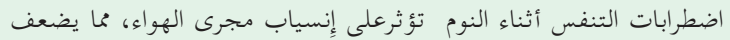

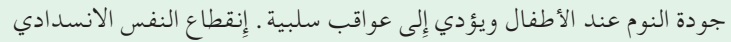

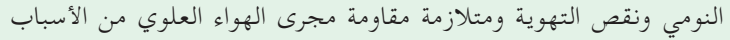

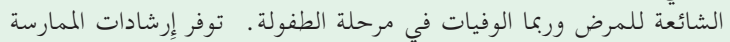

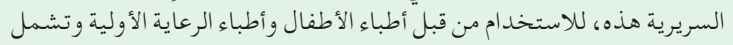

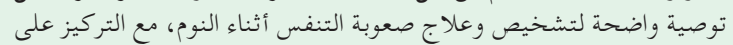

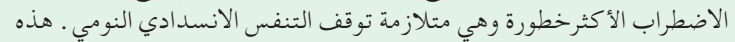

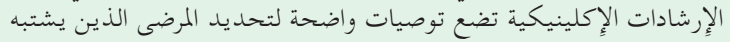

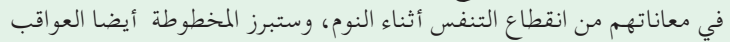

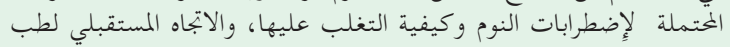
النوم عند الأطفال
\end{abstract}

Sleep-disordered breathing (SDB) includes disorders of breathing that affect airway patency, which impair children's sleep and lead to negative consequences. Obstructive sleep apnea, hypoventilation and upper airway resistance syndrome are common causes of morbidity and mortality in childhood. These clinical practice guidelines, intended for use by pediatricians and primary care clinicians, provide a clear recommendation for the diagnosis and management of sleep-disordered breathing, focusing on the most serious disorder, obstructive sleep apnea syndrome (OSAS). These clinical guidelines formulate clear recommendations to identify patients with suspected OSAS. Further, the manuscript will highlight the potential consequences of SBD in children, and how to overcome such difficulties, what could be the therapeutic options, a 12 recommendations and what are the future direction for pediatric sleep medicine.

Keywords: sleep-disordered breathing, obstructive sleep apnea syndrome (OSAS); obesity;polysomnography; adenotonsillectomy; continuous positive airway pressure (CPAP)

Saudi Med J 2020; Vol. 41 (9): 916-929 doi: $10.15537 /$ smj.2020.9.25262
From the Pediatric Respiratory and Sleep Medicine, Pediatric Department, Prince Sultan Military Medical City, Riyadh, Kingdom of Saudi Arabia.

Address correspondence and reprint request to: Dr. Abdullah Al-Shamrani, Consultant, Pediatric Respiratory and Sleep Medicine, Pediatric Department, Prince Sultan Military Medical City, Riyadh, Kingdom of Saudi Arabia. E-mail: dr.alshamrani99@gmail.com ORCID ID: https://orcid.org/0000-0001-7157-1706

$\mathrm{P}$ ediatric obstructive sleep apnea syndrome (OSAS) is common in the pediatric age group, but it differs from adult OSA in epidemiology, mechanisms of obstruction, adverse effects, diagnostic criteria and recommended treatments. ${ }^{1}$ If not identified and treated early, it will lead to multiple medical complications, including pulmonary hypertension, behavioral problems, poor school performance, metabolic syndrome, poor quality of life, and increased health care use. ${ }^{2}$ There have been significant improvements in understanding the pathophysiology of OSAS in the last 2 decades and how to diagnose and manage such conditions. The literature was reviewed using a MEDLINE search through May 2020, and a large number of articles were selected and ranked according to the best evidence. These clinical guidelines are intended to help the front-line physician, such as pediatrician, family physician or ENT physician, determine what to do in such cases. While reviewing the history of sleep in pediatric patients, the first report was dated to Dr. William Hill and describes a patient with OSAS as follows: "a stupid child who is a mouth breather and snore at night with a frequent complains of headache at school time." 3 Dr. Cristian Guilleminault in 1973 was the first physician to describe sleep apnea: "A new clinical syndrome, with repeated episodes of cessation of breathing and frequent arousal at night that leads to insomnia and tiredness next day". ${ }^{4}$ Three years later, the first description in the pediatric age group was reported. ${ }^{5}$ The American Academy of Sleep Medicine has highlighted the importance of sleep 
and suggested that a routine question should be added in any patient encounter during clerking. ${ }^{2}$ Due to the scarce of the OSA symptoms in pediatric age group, a high index of suspicions is strongly recommended, otherwise a detailed questioner is needed as screening tool for picking up such cases. Fortunately, there has been a tremendous improvement in the literature over the last 2 decades led by Dr. Gozal, who described SDB in detail as a wide spectrum of disorders with a very large impact on child health. ${ }^{6-9}$

These guidelines stress the following recommendations for the diagnosis of OSAS: 1) All children should be screened for snoring. 2) Snoring is pathological and should be investigated further. 3) OSA is common and increasing in children. 4) The prevalence of OSA is increasing. 5) OSAS requires a high level of suspicion, as symptoms in children could be scarce. 6) Detailed examination should focus on adenotonsillar disease, body mass index, and cardiac and craniofacial malformations. 7) High-risk patients or patients with comorbidities should be referred to a sleep physician. 8) High-risk patients should be monitored postoperatively. 9) Polysomnography (PSG) is the gold standard to confirm OSA and differentiate it from other types of sleep-disordered breathing. 10) Cardiac, metabolic, and neurobehavioral consequences should be carefully examined. 11) Adenotonsillectomy (AT) is the first-line while noninvasive ventilation with continuous or bi-level positive airway pressure (CPAP) or Bi Level Positive Airway Pressure (Bi-PAP) is the second-line therapy. 12) Potential residual OSA should be considered, and reevaluated for potential extra therapy.

These clinical approach is not intended to be a sole source of guidance in the evaluation of OSAS or replace clinical judgment; they are designed to assist pediatricians or primary care physicians in how to diagnose or treat such cases. In this review, we will explore all aspects related to OSA in pediatric patients, including the definition, epidemiology, pathophysiology, clinical features, morbidities, diagnosis, and treatment of OSA and end with a conclusion.

Disclosure. Authors have no conflict of interests, and the work was not supported or funded by any drug company. Dr. Adel S. Alharbi is a member of the Editorial Team, and was therefore excluded from any final editorial decisions regarding this paper.
Definition. Sleep-disordered breathing (SDB) is a clinical spectrum with frequent episodes of either complete or partial obstruction of the upper airway during sleep that ranges from snoring to apnea. Sleepdisorder breathing is classified with increasing severity as follows:

Primarysnoring. Known as simple snoring, this is the mildest form of SDB. Although known as habitual, it is defined as snoring more than 3 nights per week without evidence of hypoxia, hypercarbia or arousability. 7,10

Upper airway respiratory resistance syndrome. Defined as snoring with increased effort needed to breathe during sleep and frequent arousals, without encountered obstructive events nor abnormal change in blood gas. ${ }^{7,10}$

Obstructive hypoventilation (HV). Defined as snoring plus elevated end-expiratory carbon dioxide partial pressure in the absence of recognizable obstructive events. ${ }^{7,10}$

Obstructive sleep apnea syndrome. Defined as a disorder of breathing during sleep characterized by intermittent complete obstructive or prolonged partial upper airway obstruction and/or apnea that distort ventilation while in a sleep further disturb normal sleep patterns. ${ }^{2}$ Classification based on PSG results as proposed by Dayyat et $\mathrm{al}^{7}$ stated that it is essential to differentiate OSA from other disorders as the treatment and complications are different.

Epidemiology. Sleep-disordered breathing occurs in all ages, starting from neonates to adulthood and the prevalence varies depending on the targeted populations studied, the methodology used, and criteria used for the diagnosis. Obstructive sleep apnea syndrome is estimated to occur in $1-5 \%$ of cases, with the most predominate age occurring at 2-8 years of age, with a mean of 14 months, which could be related to the peak growth of lymphoid tissue relative to or pharyngeal size. While the prevalence of primary snoring is reported to be approximately $15 \%$, although it has been reported to be as high as $30 \%$ in the pediatric age group, the ratio of the prevalence of primary snoring to OSA vary from $4: 1$ to $6: 1 .^{7,9,11-13}$ Obstructive sleep apnea occurs equally among boys and girls during the prepubertal stage., ${ }^{914-16}$ It has been observed that environmental and family history, especially familial history of OSAS, snoring, allergies and exposure to environmental tobacco smoke, increase the risks. Prematurity also adds risk. Prevalence was reported to be higher (up to 3.5 times) in Asian and black children than in Caucasian children. From the adult literature, anthropometric characteristics of craniofacial features and racial differences could be 
predisposing factors. ${ }^{10,13,14}$ Obstructive sleep apnea is more common in certain conditions, such as Down syndrome, where the prevalence ranges from $31 \%$ to $72 \% .{ }^{17,18}$ Neuromuscular disease increases the risk of OSA. ${ }^{19,20}$ Cerebral palsy increases risk for OSA due to palatopharyngeal incoordination. ${ }^{21}$ Obstructive sleep apnea has started to be as a major health problem in obese children with an alarming very high prevalence of $33-76 \% .^{22}$

Methods. We searched PubMed up to May 2020 using the following words in different combinations: Sleep-disordered breathing, obstructive sleep apnea syndrome, obesity, PSG, AT, continuous positive airway pressure (CPAP), Bi level positive airway pressure (BiPAP), consequences, complications, adenoidectomy, health care, steroid and guideline. Search filter include publications in the last 15 years, human species, and age less than 18 years. With these search, 745 were identified. Papers were excluded based on titles and abstract. More than 150 studies were includes. The included studies where highly selected based on the

Table 1 - Disorders or conditions predisposing to obstructive sleep apnea syndrome (OSAS) in children.

Hypertrophy (adenoid, tonsil or both)
Obesity (body mass index >30)
Craniofacial abnormalities
Mandibular retrognathia
Mandibular hypoplasia (namely, Pierre Robin sequence, Treacher
Collins syndrome)
Midfacial hypoplasia (namely, craniosynostosis eg Apert syndrome,
Crouzon syndrome)
Storage disease: Hurler disease, Hunter disease or other types of
Mucopolysaccharidoses
Abnormal control of breathing
Cerebral palsy
Rapid-onset obesity, hypothalamic dysfunction, hypoventilation, and
autonomic dysregulation (ROHHAD), Joubert syndrome
Combinations of the above disorders or conditions
Down's syndrome
Sickle cell disease
Achondroplasia, hypochondroplasia
Prader-Willi syndrome
Osteopetrosis
Duchenne muscular dystrophy

best evidence available. Guidelines and Cochrane reviews were intensively reviewed. The evidence was inspired from the recommendations in the center of evidence-based medicine website (www.cebm.net).

Pathophysiology. The pathophysiology of OSAS in children still poorly understood. ${ }^{23,24}$ The etiology of OSAS is multifactorial, involving a combination of structural and neuromuscular abnormalities of the upper airway. The upper airway is made of soft tissue that easily collapses during inspiration. This change is a dynamic process involving interactions between the sleep state, the air flow and the respiratory drive. The pharyngeal lumen both length and size is best explained by the balance force theory of Remmers et al. ${ }^{24}$ In the awake condition when neuromotor systems are active, there is negative intrapharyngeal pressure during inspiration created by the diaphragm and the outward dilating action of the upper airway muscles, mainly the genioglossus, and the pharyngeal lumen remains patent. When sleep commences, the neuromuscular tone decreases, resulting in a decrease in the pharyngeal luminal cross sectional area, which results in a flow limitation. ${ }^{25,26}$ Enlarged tonsils and adenoids are clearly an important risk factor in children, and the sizes of the tonsils and adenoids increase from birth, reaching the peak of development at 2-4 years then start to subside after the age of 4 years, another surge of growth may occur in the pubertal period during growth spurt; however, a number of studies have shown that there is no absolute correlation between the size of the tonsils and adenoids and the presence of OSAS. ${ }^{26}$ Genetic factors has an important role in the pathophysiology of OSA (Table 1). ${ }^{27}$ Children with obesity are certainly at high risk for developing $\mathrm{SDB}$, and there is direct proportional between the severity of OSA to the degree of obesity, it was reported that the risk for OSA increased by $12 \%$ for any increment in body mass index (BMI) by $1 \mathrm{~kg} / \mathrm{m}^{2}$ beyond the mean BMI for age and gender. ${ }^{22}$ Obstructive sleep apnea is divided into 2 main types: type 1 involves marked lymphoid hypertrophy in the absence of obesity, while type 2 is associated with obesity and mild lymphoid hyperplasia. ${ }^{7}$

Clinical features. History. Obstructive sleep apnea syndrome can cause a variety of daytime and nighttime symptoms in children. The majority of patients with OSAS present with nighttime symptoms such as a history of loud snoring, witnessed apnea and restlessness during sleep. Parents usually describe that their children are not comfortable during sleep with extended neck, paradoxical chest movement and 
gasping, choking and awakenings from sleep, and these symptoms may leave the parents anxious as they wait around the child to shake the child with the aim of terminating these events, which leads to disturbances in the parents' sleep. Occasionally, they prefer to sleep in a sitting position, and in severe cases, rarely cyanotic spells of the face or extremities will be observed. ${ }^{28}$ Nocturnal enuresis is very pathognomonic for severe OSA and is present in $25 \%$ of patients (Table 2). ${ }^{29}$ Despite such large concerns, primarily at night, these children breathe smoothly during the daytime when awake.

Daytime symptoms depend on the underlying cause. If the main causative factor is adenotonsillar hypertrophy, the child may present with mouth breathing, poor oral hygiene, frequent upper respiratory tract infection, frequent otitis media, hyperactivity and even low attention, dysphagia and poor oral appetite, while the patient with obesity as the underlying cause may present with metabolic syndrome, excessive daytime sleepiness and mood change. ${ }^{30}$

During sleep, loud snoring, tachypnea with labored breathing, ${ }^{1,6,7,10}$ flaring alae nasi, tracheal tug and sometimes paradoxical breathing will be heard. Physicians should be alert for potential complete obstruction of the breathing passage where the child shows some respiratory effort but no snoring is heard; airflow will be detected, which might continue for several seconds, until the child is aroused and awakened, which might be followed with a sigh. The child's color might become more dusky, and the event often frightens the parents. $^{31}$

Physical examination. Usually, the physical examination is conducted while the child is awake either at the clinic or emergency dependent, and the examination might be completely normal. ${ }^{1}$ This high level of suspicion might be easily overlooked, and we used to advise parents to records the event a head of time to the doctor visit, although the majority of symptoms are apparent in the last third of the night when the parents are often in deep at sleep. ${ }^{1,30}$ It is rare for physicians to have the opportunity to see the event. Meticulous physical examination conducted on the patient with OSAS should focus on the following points: the growth chart of the patients might show failure to thrive $e^{4,32,33}$ or obesity. ${ }^{7,33}$ The presence of an adenoid face should be looked for. Craniofacial malformations (retrognathia, micrognathia, hypoplasia of maxillary bone) should be assessed..$^{34}$ The nasal passage should always be inspected for any deviation, blockage, hypertrophied nasal mucosa, or presence of secretion, the speech quality should be
Table 2 - Normal values for polysomnography. ${ }^{41,42}$

\begin{tabular}{lcc}
\hline Measurement & Children & Comment \\
\hline Total sleep time & $\geq 6$ hours & \\
Sleep efficiency & $\geq 85$ & Large variability \\
Sleep latency (minutes) & 23 & Large variability \\
Arousal index (number/ & $9-16$ & \\
hours) & & \\
REM latency & $87-155$ minutes & $136-156$ minutes \\
& $\leq 10$ years & \\
Stages (TST \%) & & \\
$\quad$ Stage N1 & $4-5$ & REars \\
Stage N2 & $44-56$ & during infancy \\
Stage N3 & $17-21$ & \\
Stage R & $\leq 1.4$ & \\
$\begin{array}{l}\text { Apnea-hypopnea index } \\
\text { (number/hours) }\end{array}$ & $\leq 29$ years \\
Central apnea index & $\leq 0.4$ & \\
$\begin{array}{l}\text { Time with saturation } \\
\text { 990 }\end{array}$ & 0 & \\
$\begin{array}{l}\text { Peak end-tidal carbon } \\
\text { dioxide ( } \geq 50 \text { mm Hg) }\end{array}$ & $\geq 25$ & \\
PLMI & & \\
\hline
\end{tabular}

REM: rapid eye movement, PLMI - periodic leg movement index, TST - total sleep time

assessed as some patients might show nasal quality, and fluids behind ear drum might be observed in the presence of adenotonsillar hypertrophy. ${ }^{34}$ The patency pharynx should be evaluated for tongue size (grades +1 [intravelic] and +2 [extravelic] are normal, while +3 [not reaching midline] and +4 [reaching midline] are enlarged), as it is commonly associated with type 1 OSA. ${ }^{26,35}$ Several studies did not show any relationship between the size of the tonsils and adenoids and the presence of OSAS. ${ }^{25,35,36}$ Generally, in the literature, there is an agreement to use history and physical examination as a screening tool to determine whether patients need extra investigation, such as a complete sleep study, but there remains some controversy regarding interventions such as adenotonsilectomy. The presence of pectus excavatum reflects long-standing disease, and accentuated heart sounds in the pulmonary area are a reflection of potential pulmonary hypertension. ${ }^{5,8,32}$ Often, chest auscultation is clear. Detailed neurological and developmental should be assessed in any patient with suspected SDB. ${ }^{37}$ 
Diagnosis. Early diagnosis and referral to optimal therapists will decrease morbidity and mortality. ${ }^{2,25}$ There is often a significant delay in the diagnosis of OSAS that could exceed 3 years due to multiple factors, including physicians, parents and third parties. The American Academy recommends starting screening patients with snoring; if they are positive, then they should be evaluated further for SDB, especially OSA. ${ }^{38}$ Polysomnography (PSG). In the literatures, PSG is the gold standard tool in the diagnosis OSA in children. It remains very expensive and time-consuming diagnostic tool, until this moment, is not easy to be conducted, no alternative method for diagnosing OSA in children. It is important to remember that the parameter used in scoring system for the respiratory events in children is totally different from those used in adults. Furthermore, PSG can differentiate primary snoring from OSA, and the severity of OSA can be determined. ${ }^{39}$

Indications for PSG: 1) Clinical presentation of OSAS; 2) Prior AT to treat OSAS; 3) Persistent symptoms after AT; 4)Assess for potential residual OSAS in high risk group children (severe OSAS, obesity, craniofacial anomalies and neurologic disorders); 5) Titration of nasal CPAP; 6) HV whether central (congenital central alveolar HV syndrome or peripheral (neuromuscular disorders or chest wall deformities); 7) Infants who have experienced a brief resolved unexplained event (BRUE); 8) Prior to decannulation in tracheostomy cases.
Diagnostic criteria for OSA by the American Academy of Sleep Medicine consist of both clinical and polysomnographic criteria, ${ }^{40,41}$ and the normal values are shown in Table 2.

Clinical criteria: presence of at least one of the following features: a) snoring; b) labored, paradoxical or obstructed breathing during sleep; c) sleepiness, hyperactivity, behavioral problems, or learning problems.

Polysomnographic criteria: 1) More than one apnea per hour of sleep (obstructive, mixed or hypopneas) or 2) Hypercarbia $>50 \mathrm{~mm} \mathrm{Hg}>25 \%$ of the total sleep time with at least one of the following: snoring or flattening of inspiratory nasal pressure waveform or paradoxical thoraco-abdominal motion.

Classification of SDB severity categories. SDB is classified with increasing severity as follows: (I) primary snoring; (II) upper airway respiratory resistance syndrome; (III) hypoventilation; (IV) obstructive sleep apnea (Table 3).

Table 4 summarized the severity of OSA based on clinical and laboratory result. ${ }^{41,42}$

$A$ home sleep apnea test (HSAT). In recent adult literatures, a home sleep apnea test (HSAT) is an acceptable alternative diagnostic tool to (PSG) in selected patient to confirm the OSA, but utility in children is not yet valid. ${ }^{43,44}$

Home PSG. Home PSG is a relatively new diagnostic tool to assess the OSA, it is less expensive than the usual PSG, and often associated with some

Table 3 - The severity of sleep-disordered breathing (SDB) based on polysomnography studies., ${ }^{7,42,43}$

\begin{tabular}{|c|c|c|c|c|}
\hline SDB based & $\begin{array}{c}\text { AHI } \\
\text { (hour/TST) }\end{array}$ & $\begin{array}{c}\text { Nadir } \\
\text { Sp02 (\%) }\end{array}$ & $\begin{array}{c}\text { PET CO } \\
>50 \mathrm{~mm} \mathrm{Hg} \\
(\% \mathrm{TST})\end{array}$ & $\begin{array}{c}\text { RAI } \\
\text { (hour/TST) }\end{array}$ \\
\hline Normal & $\leq 1$ & $>94$ & $<10$ & $<1$ \\
\hline Primary snoring & $\leq 1$ & $>94$ & $<10$ & $<2$ \\
\hline UARS & $\leq 2$ & $>92$ & $10-15$ & $\geq 2$ \\
\hline Hypoventilation & $\leq 2$ & $>92$ & $>20$ & $\geq 2$ \\
\hline \multicolumn{5}{|c|}{ Obstructive sleep apnea } \\
\hline Mild & $2-5$ & $88-92$ & $10-15$ & $2-5$ \\
\hline Moderate & $5-10$ & $80-88$ & $15-20$ & $5-8$ \\
\hline Severe & $>10$ & $<80$ & $>20$ & $>8$ \\
\hline $\begin{array}{r}\text { AHI: apnea hy } \\
\mathrm{R}\end{array}$ & $\begin{array}{l}\text { ex, } \mathrm{SPO}_{2} \text { : sat } \\
\text { y arousal ind } \\
\text { UARS: upp }\end{array}$ & $\begin{array}{l}\text { on, PET C } \\
\text { IV: hypover } \\
\text { way respira }\end{array}$ & $\begin{array}{l}\text { cutaneous end } \\
\text { n, TST: total sl } \\
\text { sistance }\end{array}$ & rbon dioxide, \\
\hline
\end{tabular}


technical difficulties as children could remove leads. Reconnection could be difficult, for this reasons, retesting might be needed further, it may underestimate the severity of OSA.

Nocturnal pulse oximetry. Overnight oximetry is increasing in popularity as a diagnostic test for OSA, as it is inexpensive, available in most centers, easily performed in hospitals or at home, and generally easily processed by computer software programs. ${ }^{45,46}$ Overnight oximetry proved to be a good alternative tool for OSA screening in the adult population. In other healthy children, overnight oximetry has a $97 \%$ positive predictive value for OSA. ${ }^{47}$ Thus, abnormal overnight oximetry test in a child with symptoms of OSA is frequently considered adequate to recommend proceeding to first-line therapy, AT. In literature, a positive result for OSA was reported when patient has $\geq 3$ desaturation clusters and at least 3 desaturations $<90 \%$ (Figure 1). Overnight pulse oximetry is useful for the OSA severity, The McGill Oximetry Score which was proposed by the Canadian Brouilette group is recommended scale in children with OSA; however, in patients with symptoms suggestive of OSA may have unremarkable overnight pulse oximetry, for this absence of nocturnal hypoxemia on home oximetry testing does not rule out a diagnosis of OSA. Approximately $40 \%$ of children with unremarkable oximetry were subsequently identified as having clinically significant OSA on PSG testing.

Figure 1 summarized the oxygen saturation distribution. Clusters of desaturation occur more frequently in the rapid eye movement (REM) stage, which is very consistent with OSA.

Nap study. Due to the difficulty of reaching the sleep center plus the long waiting lists and the expenses of this type of study, napping studies have emerged as a potential alternative method for PSG, but spontaneous daytime sleep is difficult and unfamiliar for children; furthermore, daytime sleep rarely contains the REM stage where most of the respiratory events occur, and therefore, the severity of OSA is often underestimated. Sensitivities ranged from $69 \%$ to $75 \%$ and specificities

Table 4 - Severity of obstructive sleep apnea (OSA) based on clinical and laboratory result. ${ }^{41}$

\begin{tabular}{lccc}
\hline Clinical and laboratory result & Mild & Moderate & Severe \\
\hline Growth parameter & Normal & Poor growth & FTT \\
Cardiac strain & None & $\begin{array}{c}\text { Mild ECG/ECHO } \\
\text { abnormalities }\end{array}$ & $\begin{array}{c}\text { Cor pulmonale } \\
\text { Neurological signs }\end{array}$ \\
Apnea-hypopnea index & None & Subtle & Developmental delay \\
Exhaled carbon dioxide $(\mathrm{mm} \mathrm{Hg})$ & $<5$ & $5-10$ & $>10$ \\
Saturation & $>92$ & $55-60$ & $>60$ \\
\hline
\end{tabular}

FTT - failure to thrive

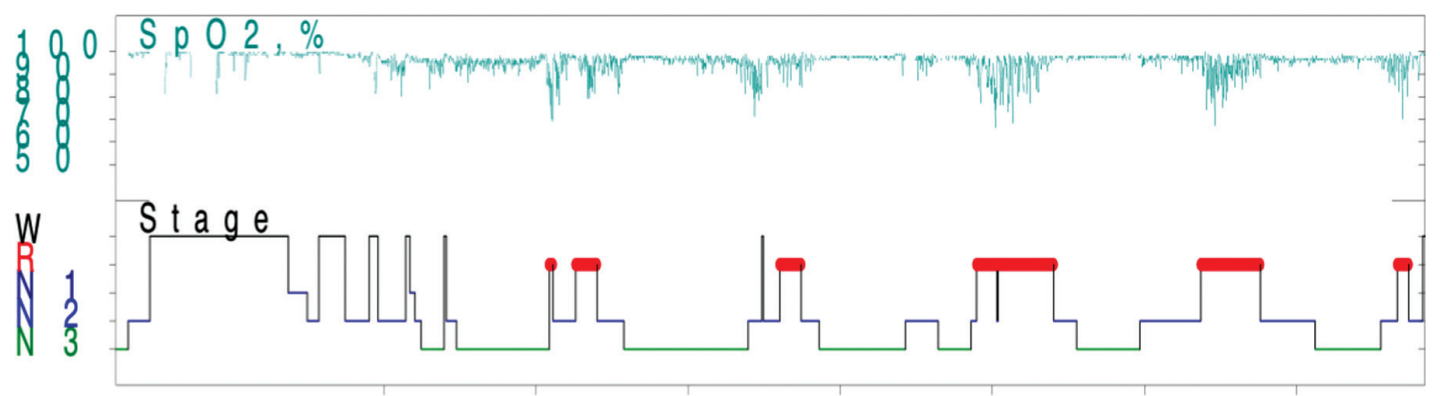

\section{$22: 14: 15 \quad 00: 0001: 0002: 0003: 0004: 0005: 0006: 00$}

Figure 1 - Oxygen saturation distribution. 
from $60 \%$ to $100 \%$ have been reported. A positive study is enough to proceed for AT, but a negative study does not rule out significant OSA because nap studies are not recommended in children. ${ }^{48}$

Audio and video taping. Audio taping at home has a $75 \%$ positive predictive value while videotaping could reach up to $83 \%$ to detect OSA in children. Both can record apneas with ease but the main negative side for these testing are underestimating hypoapnea and flow limitations. Furthermore, discrepancies from different centers make this method unreliable in children. ${ }^{49}$

Pediatric sleep questionnaire (PSQ). The PSQ is a validated questionnaire that contains 22 symptom items filled by parents prior to or on arrival to the sleep center in 4 main domains: assess snoring (frequency, and loudness), sleep related breathing (witnessed apneas, restlessness during sleep, enuresis, sleeping with extended neck,) day time sleepiness (hard to awake up, feeling unrefreshed or morning headache,) and behavioral problems (hyperactive or low attention and easily distracted). The PSQ is a useful screening tool with sensitivity of $85 \%$ and specificity of $87 \%$, in healthy children with good internal consistency and good test-retest stability. ${ }^{50}$ The PSQ can predict outcomes in children undergoing AT for SDB, and it can be utilized as screening tool to identify patients with SDB who might improve with AT (Appendix 1). ${ }^{51}$

Lateral neck $x$-ray. Lateral neck $\mathrm{x}$-ray is simple and widely used in pediatric patients with low cost and minimal radiation. Often, it does not represent the real situation as it is usually acquired with the child in the awake condition. ${ }^{52}$ Brook et $\mathrm{al}^{37}$ found a reasonable result when the adenoid to nasopharyngeal size was used in correlation with the apnea-hypoapnea index (AHI) with positive of $71 \%$ and negative predictive values of $75 \%$.

Magnetic resonance imaging. Magnetic resonance imaging of the airway is an excellent tool to identify and localize the site of obstruction for certain subgroups of children, such as those with obesity or those with craniofacial malformations. It is an excellent tool to assess the soft tissue anatomy without risk of radiation and provides beautiful pictures, whereas the disadvantages include cost, age and weight limitations, and sedation. ${ }^{53}$

Nasoscopy. Flexible fiberoptic endoscopy is a simple tool allows proper evaluation of the nasal passages. It assess the nasal patency, helps in detailed description of intranasal structures (namely, choanal atresia, nasal deviations, turbinates' hypertrophy or enlarged adenoid. It is a great tool to assess the Epiglottis to assess presence of larygomalacia or vocal cord dysfunction. In selected patients, drug-induced sleep endoscopy (DISE) is safe and helpful in assessing the severity of OSA $;{ }^{54}$ however, its use in children remains controversial. There are some promising data as this method can explore the site of obstruction especially at hypopharyngeal area and can be indicative of moderate to severe or residual OSA. ${ }^{55}$

Microbiome. Huajuan $\mathrm{Xu}$ et $\mathrm{al}^{56}$ reported potential use of oral and urinary micro biomes as alternative to PSG in diagnosing OSA especially in centers with the long waiting list for the sleep study.

\section{Consequences of sleep-disordered} breathing. Cardiovascular consequences. This has been the presentation of OSA in early literature as these cases were missed for a long time. ${ }^{32}$ Pulmonary hypertension is the most severe cardiovascular consequence if OSA is not treated. Fortunately, we are not seeing these consequences as often as before due to the early recognition of OSA and early intervention. Although, the exact cause is not clear, upper airway obstruction leading to intermittent hypoxia during sleep, such can cause increase in pulmonary artery pressures and potentially lead to abnormal control of blood pressure (BP), endothelial dysfunction and cardiac remodeling. Amin et $\mathrm{al}^{57}$ observed an alarming result (namely, obliteration of the natural reduction of blood pressure during sleep (reduced nocturnal dipping), greater variability of mean BP between both awake and sleep status, and higher systolic BP at night, such are well known risk factors for ischemic heart disease in adults. Kirk et $\mathrm{a}^{58}$ observed increased BP during sleep in 30 nonobese OSA patients in the absence of any other risk factor for high BP. Obstructive sleep apnea in children present with low level of exercise capacity led to decreased cardiac output and oxygen consumption at peak exercise capacity. ${ }^{59}$ Obstructive sleep apnea was associated with increased left ventricular mass, where abnormalities in LV geometry were present in $15 \%$ of children with primary snoring compared with 39\% of children with OSA. ${ }^{60}$ Gozal et al ${ }^{61}$ in those with a strong family history of ischemic heart disease, found significant improvement in endothelial dysfunction after treatment of OSA with AT or CPAP, suggesting that genetic and environmental factors affect the presentation. Overnight, heart rate recently emerged as a sensitive parameter to assess the severity of OSA in children as reported in CHAT study. ${ }^{45,62,63}$ Obstructive sleep apnea established as a risk for cardiovascular morbidities. Redline et $\mathrm{al}^{63}$ reported children with snoring who underwent complete sleep study found to have, 55\% are positive for OSA. Fifty-two percent of children with severe OSA had systolic BP in stage 2 when 
measured 2-3 time from right hand in sitting position in the OPD clinic. $^{64}$ The OSA affect the circadian rhythm for hormone release leading to disturbance in the hypothalamic-pituitary-adrenal axis resulting in an increment of cortisol release, which has some effect on the metabolic and BP regulation. ${ }^{65}$ Marcus et $\mathrm{al}^{66}$ studied 41 children with OSA were predominant male $66 \%$ further, $32 \%$ were hypertensive. Nowadays, severe OSA in children increasing mean pulmonary artery pressure and right ventricular dysfunction. ${ }^{67}$ Prevalence rates of cor pulmonale and congestive heart failure decreased after AT but have been reported to be 10-12\%.4,45,68

Neurometabolic. Neurocognitive dysfunction is now a well-recognized consequence of OSA in children. ${ }^{4,7}$ Obstructive sleep apnea has been reported to cause significant school problems in children, such as low attention, aggressive behavior, poor academic performance, excessive daytime sleepiness and behavioral illness. ${ }^{69}$ Obstructive sleep apnea-type 1 has been associated with hyperactivity and with impaired cognitive function. ${ }^{70-72}$ Approximately $25 \%$ of those with OSA exhibit ADHD; on the other hand, 70\% of those with ADHD may show signs of OSA, and this behavior improved after AT. ${ }^{72,74}$ Low levels of attention has been reported in OSA patients, and the exact mechanism remains obscure, but intermittent hypoxia and sleep fragmentation may lead to changes in neurotransmitters in the cortex, resulting in such dysfunction. ${ }^{75,76}$ Lower scoring in both (math and reading) were observed in overweight children compared to none overweight children, as increasing BMI is not favorable for cognitive function. ${ }^{77}$

In China, $\mathrm{Li}$ et $\mathrm{al},{ }^{78}$ reported that the IQ was surprisingly lower in obese children compared those with normal weight, which raised a question, can we use the BMI as a marker for academic performance. The prevalence of behavioral and learning difficulties is a dose dependent to rapid gain weight in children. The childhood adenotonsillectomy trial (CHAT) study need to be watched as it is looking not only for sleep improvement but also for neurocognitive and quality of life after the intervention. ${ }^{79}$

Growth impairment. In the earlier literature, failure to thrive was a common manifestation of OSA, and it was reported to be as $56 \%$ in patients with OSA. ${ }^{4,80}$ Luckily, the prevalence of growth impairments has been markedly reduced in recent literature. Furthermore, children often show significant improvements in weight after AT, indicating potential causative factors for growth impairment (namely, decrease in appetite and alterations in smelling, increased basal metabolic rate due to stridorous and labored breathing, dysphagia due to enlarged tonsils, decreased insulin growth factor-1 and growth hormone release). In fact, weight gain remained a big concern for obese and non-obese children post AT. Obstructive sleep apnea should be included in the differential diagnosis in any patient with unexplained failure to thrive or poor weight gain. ${ }^{24,81}$

Obesity. Although obesity predisposes children to sleep-related upper airway obstruction, majorities of patients with OSAS are not obese. A study of OSAS in obese Singapore children estimated the prevalence to be $5.7 \%$ overall and $13.3 \%$ in morbidly obese children (percentage ideal body weight $>180$ ). ${ }^{82}$ But it was reported as high as $60 \%$ in obese children and adolescents. ${ }^{83}$ Redline et al, confirm that the obesity is a significant risk factor for OSAS in children ${ }^{63}$ Rosen reported more referrals for OSA assessment in obese children compared to nonobese children. ${ }^{84}$ Currently, the prevalence of obesity has markedly increased worldwide, for this reason a second phenotypes of OSA, similar to the adult emerged in children called OSA (type II), ${ }^{7}$ that are newly emerging, and these obese patients are prone to metabolic syndrome and decreased quality of life compared to nonobese adults. ${ }^{85}$ Fifty-five percent of children scheduled for sleeve surgery had OSA. ${ }^{86}$ The severity of OSA paralleled the severity of obesity in an analysis that suggested for each unit increase in BMI, there was a $12 \%$ higher risk of OSA. ${ }^{26}$

Metabolic syndrome. Metabolic syndrome is defined as a tetrad (obesity, dyslipidemia, insulin resistance, and hypertension). It is a known risk factor for cardiovascular disease in adults. ${ }^{87}$ Weiss et al ${ }^{88}$ found that obesity was the main component contributing to increased risk as BMI increased. Metabolic syndrome reported as 50\% in children with severe obesity. Increased BMI and raised fasting insulin levels during childhood emerging as the strongest predictors of metabolic syndrome in adulthood. In adult patients, OSA similar to obesity has been recognized as an important risk factor for metabolic syndrome ${ }^{89}$ In children, both insulin resistance (measured by the insulin/glucose ratio or clinically by presence of darkening of the skin in folds and creases, like the neck and armpits [acanthosis nigricans] and altered lipidemia [evidence of increased plasma triglyceride) are mainly affected by obesity and are less likely to be affected by OSA. However, similar to adults, when obesity and OSA-(type II) are present together the risk of metabolic syndrome will be exaggerated and may cause even liver steatosis. ${ }^{90}$

Nocturnal enuresis. Obstructive sleep apnea is associated with a higher prevalence of nocturnal enuresis, reaching $50 \%{ }^{29,32,91}$ Nocturnal enuresis has a 
direct correlation with the AHI and has been reported as the most predictive symptom for OSA in children who snore. This may be due to the effects of elevated B-type natriuretic peptide (BNP) levels that affect the renin-angiotensin pathway, where inhibiting the antidiuretic hormone leads to enuresis with significant improvement after AT. ${ }^{8}$ Obstructive sleep apnea is an important cause of nocturnal enuresis in children and should be considered in the differential diagnosis of nocturia. ${ }^{92,93}$

Malignancy. There is recent epidemiological data suggesting that OSA is carcinogenic, the potential existence of severe OSA and cancer has gained increasing attention in the last decade. ${ }^{94}$ The mechanism is not yet clear, multiple studies still going. Animal exposed to chronic intermittent hypoxia and sleep fragmentation can trigger oxidative stress and chronic inflammation, enhance oncogenic pathway, the data here still primitive and need to be observed. ${ }^{95,96}$

Health care burden. Obstructive sleep apnea is a chronic condition that affects daily life. Tarasiuk \& Reuveni ${ }^{97}$ reported $226 \%$ increased health care costs in children with OSA, and another report with a similar finding of $215 \%$ was reported by Tarasiuk \& Reuveni where the contributing factors were multiple admissions and frequent emergency visits. The Sleep Heart Health Study reported that OSA patients had an $18 \%$ increase in predicted healthcare utilization based on medication use. ${ }^{98}$ Patients with OSA have multiple additional comorbidities, and OSAS severity correlates directly to total annual costs. The treatment of OSA in children decreased health care costs by $1 / 3$ and decreased admissions after AT by $60 \% .{ }^{99}$

Management. Adenotonsillectomy remains the treatment of choice in other healthy children, and the presence of additional risk factors is not a contraindication to AT. The success rate could reach $80 \%$ in non-high-risk groups, but the patients should be observed postoperatively for potential pulmonary compromise. ${ }^{100}$ Multiple risk factors have been reported in the literature:

1. Young age: $<3$ years $^{100}$

2. Severe OSA by PSG (AHI $>10 /$ hours)

3. Associated medical conditions

- Syndromes or craniofacial anomalies ${ }^{4,102}$

- Neuromuscular disorders ${ }^{4,102}$

- Ongoing respiratory infection

- Premature birth ${ }^{4,102}$

4. Comorbidities:

- Pulmonary hypertension/cor-pulmonale $e^{4,102}$
- Failure to thrive $e^{4,102}$

- Obesity

Following surgery, patients in the category of high risk should be observed for one night in a facility where appropriate monitoring for vital signs, saturation, and proper care like noninvasive or invasive support if needed.

Residual OSA is suspected in children with comorbidities, for example, approximately $50 \%$ in obese children and 30\% in Down syndrome; these patients should be reassessed. Repeated PSG is recommended several weeks after surgery. ${ }^{100-102}$

Adenoidectomy. Adenoidectomy alone was primarily used as a first-line therapy for OSA due to the risk of morbidity, lower cost and shorter hospitalization, with relatively comparable postoperative results in children with moderate OSA, namely, AHI $<10 /$ hours, and who were not obese. ${ }^{101.102}$ The majority of researchers are not recommending adenoidectomy alone for the treatment of OSA in children because symptoms and signs of OSA frequently persist after adenoidectomy alone. ${ }^{103,104}$ Furthermore, tonsillectomy alone is less effective than AT for pediatric OSA. ${ }^{104}$

Positive airway pressure treatment. Continuous positive airway pressure is the second-line therapy in children, in contrast to adults, where it is the first-line therapy; immediate and long term efficacy are well established in adult literatures since its invention in 1983 by $\operatorname{Dr}$ Sullivan. ${ }^{105}$ Continuous positive airway pressure is a noninvasive method of providing distending pressure applied at nose then directed through the airway leading to increase airway patency during respiration and prevent soft tissue from collapse. There are several modalities for delivering the CPAP: i) Nasal: small and appropriate mask over the nose only. ii) Nasopharyngeal (NP) CPAP: an airway tube placed through the nose whose tip terminates in the nasopharynx. iii) Face mask: appropriate face mask is placed over the nose and mouth with good seal.

Continuous positive airway pressure stent the airway, aid in oxygenation through increasing the surface area of the alveolus, improve the functional residual lung capacity and decrease the effort needed to breathe during sleep. According to the American Academy of Pediatrics, it is an optional therapy in severe OSA patients until surgery takes place, and for residual OSA after AT; it has been reported to be as effective as $90 \%$ with high dropout (50-80\%). Regular follow-up is crucial. There is a need for different mask sizes to fit the children. The compliance remains a big problem and should be monitored carefully such can be achieved by behavioral techniques (training parents, positive rewards, 
desensitization, and remodeling). The child often needs several nights with continuous education until the mask is tolerated and the child feels an improvement in sleep quality. Auto CPAP is relatively new machine, have 2 setting pressure automatically adjust the airway pressure during sleep. If the patient remained symptomatic with the retention of carbon dioxide, Bi-PAP is recommended ideally through a titration study in the sleep center. Complications of noninvasive ventilation include nasal sore, nasal congestion, conjunctivitis, air leak, abdominal distension, and midfacial hypoplasia. ${ }^{106}$

Medical therapies. Children with mild or moderate OSA with blocked nose or adenoid hypertrophy are usual candidates for medical therapies as alternatives or adjuvants to AT. Different regimens have been used in children such as inhaled steroids, montelukast, or combinations

Intranasal corticosteroids. Numerous studies have proven the efficacy of inhaled steroids in patients with OSAS. It is the most effective medical therapy and often prescribed as topical intranasal corticosteroids for 4-8 weeks. ${ }^{107}$

Leukotriene modifier therapy. Montelukast for 3 months has modest benefit in the treatment of mild and moderate OSA, and it proved to be effective in reducing adenoid hypertrophy and improving OSA symptoms. ${ }^{108}$ Parents should be aware of recent concern on neuropsychiatric event in children with the use of montelukast, the data come from asthmatic patients but to be mentioned and observed. ${ }^{106,109,110}$

Combination therapy. The combination of inhaled steroids and antileukotiene for 3 months were effective in several papers. Kheirandish et $\mathrm{al}^{111}$ demonstrated an efficacy of more than $80 \%$ in mild OSA patients, especially in young and nonobese patients, in a large study with 752 patients. Occasionally, short systemic steroids might be helpful. More data still needed for long-term efficacy and safety in children using combined therapies.

Adjunct therapies. i) Weight loss is strongly recommended for obese children with OSA, it often work as adjuvant therapy. Nutritional counseling and close follow-up are recommended, especially for overweight or obese patients. ${ }^{9}$ ii) Environmental controls-children with OSA should avoid exposure tobacco smoke pollutions or indoor irritant or any other environmental allergens, as they may lead to blocked nose and the worsening of OSA symptoms. iii) Antibiotics are not routinely indicated in treatment of pediatric OSA. Few reports have shown some benefit in reducing adenotonsillar size, which might be beneficial if OSA symptoms are related to chronic sinus infection with blocked or purulent nasal discharge. ${ }^{112}$ iv) Orthodontics rapid maxillary expansion is rarely used in the pediatric age range. ${ }^{113}$ v) Other surgical options in treating OSA (although rarely needed in children) as nasal turbinate reduction, tongue reduction, tongue suspension procedure, and tracheostomy are options in some difficult cases. ${ }^{114,115}$

In conclusion, early diagnosis and treatment of pediatric OSAS is encouraged and may improve a child's long-term cognitive and social potential and school performance. Increasing prevalence of OSA type II parallels the increasing prevalence obesity and is a nightmare for sleep physicians, who expect more residual OSA and a shift in the consequences to metabolic and cardiac consequences. The country has an urgent need for effective educational programs to alert populations about the importance of the consequences of OSA and the economic burden on the health system. The utilization of sleep physicians and sleep centers is still underdeveloped. Sleep medicine is an area for future research and should be encouraged.

Acknowledgements. Authors would like to thanks Dr Mona Philby for revising the manuscript.

\section{References}

1. Carole L. Marcus and Gerald M. Loughlin. Obstructive sleep apnea in children. Seminars in Pediatric Neurology 1996; 3: 23-28.

2. Section on Pediatric Pulmonology, Subcommittee on Obstructive Sleep Apnea Syndrome. American Academy of Pediatrics. Clinical practice guideline: diagnosis and management of childhood obstructive sleep apnea syndrome. Pediatrics 2002; 109: 704-712.

3. Hill W. On some causes of backwardness and stupidity in children: And the relife of these symptoms in some instances by naso-pharyngeal scarifications. Br Med J 1889; 2: 711-712.

4. Guilleminault C, Eldridge FL, Dement WC. Insomnia with sleep apnea: a new syndrome. Science 1973; 181: 856-858.

5. Guilleminault C, Eldridge FL, Simmons FB, Dement WC. Sleep apnea in eight children. Pediatrics 1976; 58: 23-30.

6. Kheirandish-Gozal L, Gozal D, editors. Sleep disordered breathing in children. A comprehensive clinical guide to evaluation and treatment. New York (NY): Springer Science; 2012.

7. Ehab Dayyat, Leila Kheirandish-Gozal, and David Gozal, Childhood obstructive sleep apnea: One or two distinct disease entities? Sleep Med Clin 2007; 2: 433-444.

8. Capdevila OS, Kheirandish-Gozal L, Dayyat E, Gozal D. Pediatric obstructive sleep apnea: complications, management, and long-term outcomes. Proc Am Thorac Soc 2008; 5: 274-282.

9. Marcus CL, Brooks LJ, Draper KA, Gozal D, Halbower $\mathrm{AC}$, Jones J, et al. Diagnosis and management of childhood obstructive sleep apnea syndrome. Pediatrics 2012; 130: 714-755. 
10. Savini S, Ciorba A, Bianchini C, Stomeo F, Corazzi V, Vicini C, Pelucchi S. Assessment of obstructive sleep apnoea (OSA) in children: an update. Acta Otorhinolaryngol Ital 2019; 39: 289-297.

11. Ferreira AM, Clemente V, Gozal D, et al. Snoring in Portuguese primary school children. Pediatrics 2000; 106: 64.

12. O'Brien LM, Holbrook CR, Mervis CB, et al. Sleep and neuro behavioral characteristics in 5-7-year old hyperactive children. Pediatrics 2003; 111: 554-563.

13. Urschitz MS, Guenther A, Eitner S, et al. Risk factors and natural history of habitual snoring. Chest 2004; 126: 790-800.

14. Ersu R, Arman AR, Save D, et al. Prevalence of snoring and symptoms of sleep-disordered breathing in primary school children in Istanbul. Chest 2004; 126: 19-24.

15. Rosen CL, Larkin EK, Kirchner HL, et al. Prevalence and risk factors for sleep-disordered breathing in 8- to 11-year-old children: association with race and prematurity. $J$ Pediatr 2003; 142: 383-389.

16. Bixler EO, Vgontzas AN, Lin HM, et al. sleep disordered breathing in children in a general population sample: prevalence and risk factors. Sleep 2009; 32: 731-736.

17. Li AM, So HK, Au CT, et al. Epidemiology of obstructive sleep apnoea syndrome in Chinese children: a two-phase community study. Thorax 2010; 65: 991-997.

18. Marcus CL, Keens TG, Bautista DB, von Pechmann WS, Ward SL. Obstructive sleep apnea in children with Down syndrome. Pediatrics 1991; 88: 132-139.

19. Stebbens VA, Dennis J, Samuels MP et al; sleep related upper airway obstruction in a cohort's with Down syndrome. Arch Dis Child 1991; 66: 1333-1338.

20. Kerr SL, Kohrman MH, Polysomnographic abnormalities in Duchene muscular dystrophy. J Child Neurol 1994; 9: 332-334.

21. Kotagal S, Gibbons VP, Stith JA, Sleep abnormalities in patient with severe cerebral palsy. Dev Med Child Neurol 1994; 36: 304-311.

22. Kalra $M$, Inge $T$, Garcia V, et al. Obstructive sleep apnea in extremely overweight adolescents undergoing bariatric surgery. Obes Res 2005; 13: 1175-1179.

23. Katz ES, D’Ambrosio CM. Pathophysiology of pediatric obstructive sleep apnea. Proc Am Thorac Soc 2008; 5: 253-262.

24. Remmers JE, deGroot WJ, Sauerland EK, Anch AM. Pathogenesis of upper airway occlusion during sleep. J Appl Physiol 1978; 44: 931-938.

25. Laura M Sterni, David E. Tunkel, obstructive sleep apnea in children an update. Pediatr N Clinc Am 2003; 50: 427-443.

26. Wang J, Zhao Y, Yang W, Shen T, Xue P, Yan X, et al. Correlations between obstructive sleep apnea and adenotonsillar hypertrophy in children of different weight status. Sci Rep 2019; 9: 11455.

27. Redline S, Tishler PV, Schluchter M, et al. Risk factors for sleep-disordered breathing in children. Associations with obesity, race, and respiratory problems. Am J Respir Crit Care Med 1999; 159: 1527-1532.

28. Rosen CL. Clinical features of obstructive sleep apnea hypoventilation syndrome in otherwise healthy children. Pediatr Pulmonol 1999; 27: 403-409.

29. Cinar U, Vural C, Cakir B, et al. Nocturnal enuresis and upper airway obstruction. Int J Pediatr Otorhinolaryngol 2001; 59: 115-118.
30. Landau YE, Sari Greenberg-Dotan et al. Impaired behavioral and neurocognitive function in preschool children with obstructive sleep apnea. Pediatr Pulmonol 2012; 47: 180-188.

31. Katz ES, Mitchell RB, D’Ambrosio CM. Obstructive Sleep Apnea in Infants. Am J Respir Crit Care Med 2012; 185: 805-816.

32. Guilleminault C, Korobkin R, Winkle R. A review of 50 children with obstructive sleep apnea syndrome. Lung 1981; 159: 275-287.

33. Andersen IG, Holm JC, Homøe P. Obstructive sleep apnea in obese children and adolescents, treatment methods and outcome of treatment - A systematic review. Int J Pediatr Otorhinolaryngol 2016; 87: 190-197.

34. Lam DJ, Jensen CC, Mueller BA, Starr JR, Cunningham ML, Weaver EM. Pediatric sleep apnea and craniofacial anomalies: a population-based case-control study. Laryngoscope 2010; 120: 2098-2105.

35. Li AM, Wong E, Kew J, Hui S, Fok TF. Use of tonsil size in the evaluation of obstructive sleep apnoea. Arch Dis Child 2002; 87: 156-159.

36. Brooks LJ, Stephens BM, Bacevice AM. Adenoid size is related to severity but not the number of episodes of obstructive apnea in children. J Pediatr 1998; 132: 682-686.

37. Carroll JL, McColley SA, Marcus CL, et al. Inability of clinical history to distinguish primary snoring from obstructive sleep apnea syndrome in children. Chest 1995; 108: 610-618.

38. Goldstein NA, Sculerati N, Walsleben JA, et al. Clinical diagnosis of pediatric obstructive sleep apnea validated by polysomnography. Otolaryngol Head Neck Surg 1994; 111: 611-617.

39. Aurora RN, Zak RS, Karippot A, Lamm CI, Morgenthaler TI, Auerbach SH, et al. Practice parameters for the respiratory indications for polysomnography in children. Sleep 2011; 34: 379-388.

40. American Academy of Sleep Medicine. International Classification of Sleep Disorders - Third Edition (ICSD-3) (Online). 3rd ed. Darien (IL): American Academy of Sleep Medicine; 2014.

41. Marcus CL, Omlin KJ, Basinki DJ, Bailey SL, Rachal AB, Von Pechmann WS, et al. Normal polysomnographic values for children and adolescents. Am Rev Respir Dis 1992; 146: 1235-1239.

42. Uliel S, Tauman R, Greenfeld M, Sivan Y. Normal polysomnographic respiratory values in children and adolescents. Chest 2004; 125: 872-878.

43. Kapoor M, Greenough G. Home sleep tests for obstructive sleep apnea (OSA). J Am Board Fam Med 2015; 28: 504-509.

44. Rosen IM, Kirsch DB, Chervin RD, Carden KA, Ramar K, Aurora RN, et al. Clinical use of a home sleep apnea test: An American Academy of Sleep Medicine Position Statement. J Clin Sleep Med 2017; 13: 1205-1207.

45. Dehlink E, Tan HL.Update on paediatric obstructive sleep apnoea. J Thorac Dis 2016; 8: 224-235.

46. Nixon GM, Brouillette RT. Diagnostic techniques for obstructive sleep apnoea: is polysomnography necessary? Paediatr Respir Rev 2002; 3: 18-24.

47. Brouillette RT, Morielli A, Leimanis A, Waters KA, Luciano R, Ducharme FM. Nocturnal pulse oximetry as an abbreviated testing modality for pediatric obstructive sleep apnea, McGill University, Montreal. Pediatrics 2000; 105: 405-412. 
48. Saeed MM, Keens TG, Stabile MW, Bolokowicz J, Davidson Ward SL. Should children with suspected obstructive sleep apnea syndrome and normal nap sleep studies have overnight sleep studies? Chest 2000; 118: 360-365.

49. Lamm C, Mandeli J, Kattan M. Evaluation of home audiotapes as an abbreviated test for obstructive sleep apnea syndrome (OSAS) in children. Pediatr Pulmonol 1999; 27: 267-272.

50. Chervin RD, Hedger K, Dillon JE, Pituch KJ. Pediatric sleep questionnaire (PSQ): validity and reliability of scales for sleep-disordered breathing, snoring, sleepiness, and behavioral problems. Sleep Med 2000; 1: 21-31.

51. Chan BC, Galland BC, Smith LA, Maessen SE, Haszard JJ, Schaughency EA, et al Can sleep questionnaires predict outcome in children undergoing adenotonsillectomy for sleep disordered breathing? Australian Journal of Otolaryngology 2019; 2: 6.

52. Slaats MA, Van Hoorenbeeck K, Van Eyck A, Vos WG, De Backer JW, Boudewyns A, et al. Upper airway imaging in pediatric obstructive sleep apnea syndrome. Sleep Med Rev 2015; 21: 59-71.

53. Fleck RJ, Shott SR, Mahmoud M, Ishman SL, Amin RS, Donnelly LF. Magnetic resonance imaging of obstructive sleep apnea in children. Pediatr Radiol 2018; 48: 1223-1233.

54. Campanini A, Canzi P, De Vito A, Dallan I, Montevecchi F, Vicini C. Awake versus sleep endoscopy: personal experience in 250 OSAHS patients. Acta Otorhinolaryngol Ital 2010; 30: 73-77.

55. Friedman NR, Parikh SR, Ishman SL, Ruiz AG, El-Hakim H, Ulualp SO, et al. The current state of pediatric drug-induced sleep endoscopy. Laryngoscope 2017; 127: 266-272.

56. Xu H, Li X, Zheng X, Xia Y, Fu Y, Li X, et al. Pediatric obstructive sleep apnea is associated with changes in the oral microbiome and urinary metabolomics profile: A pilot study. J Clin Sleep Med 2018; 14: 1559-1567.

57. Amin R, Somers VK, McConnell K, et al. Activity adjusted 24-hour ambulatory blood pressure and cardiac remodeling in children with sleep disordered breathing. Hypertension 2008; 5: 84-91.

58. Kirk V, Midgley J, Giuffre M, Ronksley P, Nettel-Aguirre A, Al-Shamrani A. Hypertension and obstructive sleep apnea in Caucasian children. World J Cardiol 2010; 2: 251-256.

59. Evans CA, Selvadurai H, Baur LA, Waters KA. Effects of obstructive sleep apnea and obesity on exercise function in children. Sleep 2014; 37: 1103-1110.

60. Amin RS, Kimball TR, Bean JA, Jeffries JL, Willging JP, Cotton RT, et al. Left ventricular hypertrophy and abnormal ventricular geometry in children and adolescents with obstructive sleep apnea. Am J Respir Crit Care Med 2002; 165: 1395-1399.

61. Gozal D, Kheirandish-Gozal L, Serpero LD, Sans Capdevila O, Dayyat E. Obstructive sleep apnea and endothelial function in school aged nonobese children: effect of adenotonsillectomy. Circulation 2007; 116: 2307-2314.

62. Quante M, Wang R, Weng J, Rosen CL, Amin R, Garetz SL, et al. The effect of adenotonsillectomy for childhood sleep apnea on cardiometabolic measures. Sleep 2015; 38: 1395-1403.

63. Redline S, Amin R, Beebe D, Chervin RD, Garetz SL, Giordani B, et al. The Childhood Adenotonsillectomy Trial (CHAT): a Standard Surgical Procedure in a Pediatric Population. Sleep 2011; 34: 1509-1517.
64. Hinkle J, Connolly HV, Adams HR, Lande MB. Severe obstructive sleep apnea in children with elevated blood pressure. J Am Soc Hypertens 2018; 12: 204-210.

65. Späth-Schwalbe E, Gofferje M, Kern W, Born J, Fehm HL. Sleep disruption alters nocturnal ACTH and cortisol secretory patterns. Biol Psychiatry 1991; 29: 575-584.

66. Marcus CL, Greene MG, Carroll JL. Blood pressure in children with obstructive sleep apnea. Am J Respir Crit Care Med 1998; 157 (4 Pt 1): 1098-1103.

67. Sardon O, Corcuera P, Castańos L, Rezola E, Eizmendi M, Izquierdo MA, et al. Severe sleep obstructive apnea in children. Can respiratory polygraphy estimate pulmonary pressure and right heart function? European Respiratory Journal 2017; 50: 3336.

68. Lee PC, Hwang B, Soong WJ, Meng CC. The specific characteristics in children with obstructive sleep apnea and cor pulmonale. Scientific World Journal 2012; 2012: 757283.

69. Smith DL, Gozal D, Hunter SJ, Philby MF, Kaylegian J, Kheirandish-Gozal L. Impact of sleep disordered breathing on behaviour among elementary school-aged children: a crosssectional analysis of a large community-based sample. Eur Respir J 2016; 48: 1631-1639.

70. Urschitz MS, Eitner S, Guenther A, Eggebrecht E, Wolff J, Urschitz-Duprat PM, et al. Habitual snoring, intermittent hypoxia, and impaired behavior in primary school children. Pediatrics 2004; 114: 1041-1048.

71. Yewen Shi, Huanan Luo, Haiqin Liu, Jin Hou et al. International Journal of Pediatric Otorhinolaryngology 2019; 16:38-42.

72. Bourke R, Anderson V, Yang JS, et al. Cognitive and academic functions are impaired in children with all severities of sleepdisordered breathing. Sleep Med 2011; 12: 489-96.

73. O’Brien LM, Goal D. Sleep in children with attention deficit/ hyperactivity disorder. Minerva Pediatr 2004; 56: 585-601.

74. Montgomery-Downs HE, Crabtree VM, Gozal D. Cognition, sleep and respiration in at-risk children treated for obstructive sleep apnoea. Eur Respir J 2005; 25: 336-342.

75. Bass JL, Corwin M, Gozal D, Moore C, Nishida H, Parker $S$, et al. The effect of chronic or intermittent hypoxia on cognition in childhood: a review of the evidence. Pediatrics 2004; 114: 805-816.

76. Halbower AC, Degaonkar M, Barker PB, Earley CJ, Marcus CL, Smith PL, et al. Childhood obstructive sleep apnea associates with neuropsychological deficits and neuronal brain injury. PLoS Med 2006; 3: 301.

77. Datar A, Sturm R, Magnabosco JL. Childhood overweight and academic performance: national study of kindergartners and first-graders. Obes Res 2004; 12: 58-68.

78. Li X. A study of intelligence and personality in children with simple obesity. Int J Obes Relat Metab Disord 1995; 19: 355-357.

79. Ahlqvist-Rastad J, Hultcrantz E, Melander H, Svanholm H. Body growth in relation to tonsillar enlargement and tonsillectomy. Int J Pediatr Otolaryngol 1992; 24: 55-56.

80. Smith DF, Amin RS. OSA and cardiovascular risk in pediatrics. Chest 2019; 156: 402-413.

81. Roemmich JN, Barkley JE, D’Andrea L, Nikova M, Rogol $\mathrm{AD}$, Carskadon MA, et al. Increases in overweight after adenotonsillectomy in overweight children with obstructive sleep-disordered breathing are associated with decreases in motor activity and hyperactivity. Pediatrics 2006; 117 : 200-208. 
82. Chay OM, Goh A, Abisheganaden J, Tang J, Lim WH, Chan YH, et al. Obstructive sleep apnea syndrome in obese Singapore children. Pediatr Pulmonol 2000; 29: 284-290.

83. Verhulst SL, Van Gaal L, De Backer W, Desager K. The prevalence, anatomical correlates and treatment of sleepdisordered breathing in obese children and adolescents. Sleep Med Rev 2008; 12: 339-346.

84. Rosen CL. Clinical features of obstructive sleep apnea hypoventilation syndrome in otherwise healthy children. Pediatr Pulmonol 1999; 27: 403-409.

85. Friedlander SL, Larkin EK, Rosen CL, Palermo TM, Redline S. Decreased quality of life associated with obesity in school-aged children. Arch Pediatr Adolesc Med 2003; 157: 1206-1211.

86. Kalra M, Inge T, Garcia V, Daniels S, Lawson L, Curti R, Cohen A, Amin R. Obstructive sleep apnea in extremely overweight adolescents undergoing bariatric surgery. Obesity Research 2005; 13: 1175-1179.

87. Tresaco B, Bueno G, Pineda I, Moreno LA, Garagorri JM, Bueno M. Homeostatic model assessment (HOMA) index cut-off values to identify the metabolic syndrome in children. J Physiol Biochem 2005; 61: 381-388.

88. Weiss R, Kaufman FR. Metabolic complications of childhood obesity. Diabetes Care 2008; 2: 310-316.

89. Punjabi NM, Sorkin JD, Katzel LI, Goldberg AP, Schwartz AR, Smith PL. Sleep-disordered breathing and insulin resistance in middle aged and overweight men. Am J Respir Crit Care Med 2002; 165: 677-682.

90. Tauman R, O’Brien LM, Ivanenko A, Gozal D. Obesity rather than severity of sleep-disordered breathing as the major determinant of insulin resistance and altered lipidemia in snoring children. Pediatrics 2005; 116: 66-73.

91. Su MS, Xu L, Pan WF, Li CC. al. Current perspectives on the correlation of nocturnal enuresis with obstructive sleep apnea in children. World J Pediatr 2019; 15: 109-116.

92. Jeyakumar A, Rahman SI, Armbrecht ES, Mitchell R. The association between sleep-disordered breathing and enuresis in children. Laryngoscope 2012; 122: 1873-1877.

93. Mehta B, Waters K, Fitzgerald D, Badawi N. Sleep disordered breathing $(\mathrm{SDB})$ in neonates and implications for its long-term impact. Paediatr Respir Rev 2020; 34: 3-8.

94. Owens RL, Gold KA, Gozal D, Peppard PE, Jun JC, Dannenberg AJ, et al. Sleep and Breathing ... and Cancer? Cancer Prev Res (Phila) 2016; 9: 821-827.

95. Sillaha A, Nathaniel F. Watson NF, Gozal D, Phipps AI. Obstructive sleep apnea severity and subsequent risk for cancer incidence. Prev Med Rep 2019; 15: 100886.

96. Santamaria-Martos F, Benítez I, Girón C, Barbé F, MartínezGarcía MA, Hernández L, et al. Biomarkers of carcinogenesis and tumour growth in patients with cutaneous melanoma and obstructive sleep apnoea. Eur Respir J 2018; 51: 1701885.

97. Tarasiuk A, Reuveni H. The economic impact of obstructive sleep apnea. Curr Opin Pulm Med 2013; 19: 639-644.

98. Tarasiuk A, Greenberg-Dotan S, Simon-Tuval T, et al. Elevated morbidity and healthcare use in children with obstructive sleep apnea syndrome. Am J Respir Crit Care Med 2007; 175: 55-51.

99. Brietzke SE, Gallagher D. The effectiveness of tonsillectomy and adenoidectomy in the treatment of pediatric obstructive sleep apnea/hypopnea syndrome: a meta-analysis. Otolaryngol Head Neck Surg 2006; 134: 979-984.
100. Rosen GM, Muckle RP, Mahowald MW, Goding GS, Ullevig C. Postoperative respiratory compromise in children with obstructive sleep apnea syndrome: can it be anticipated? Pediatrics 1994; 93: 784-788.

101. Wilson K, Lakheeram I, Morielli A, Brouillette R, Brown K. Can assessment for obstructive sleep apnea help predict postadenotonsillectomy respiratory complications? Anesthesiology 2002; 96: 313-322.

102. Biavati MJ, Manning SC, Phillips DL. Predictive factors for respiratory complications after tonsillectomy and adenoidectomy in children. Arch Otolaryngol Head Neck Surg 1997; 123: 517-521.

103. Baugh RF, Archer SM, Mitchell RB, Rosenfeld RM, Amin R, Burns JJ, et al. Clinical Practice Guideline: Tonsillectomy in Children. Otolaryngol Head Neck Surg 2011; 144: 1-30.

104. Domany KA, Dana E, Tauman R, Gut G, Greenfeld M, Yakir $\mathrm{BE}$, Adenoidectomy for obstructive sleep apnea in children. Clin Sleep Med 2016; 12: 1285-1291.

105. Sullivan CE, Berthon-Jones M, Issa FG. Remission of severe obesity-hypoventilation syndrome after short-term treatment during sleep with nasal continuous positive airway pressure. American Review of Respiratory Disease 1983; 128: 177-181.

106. Booth C, Premkumar MH, Yannoulis A, et al. Sustainable use of continuous positive airway pressure in extremely preterm infants during the first week after delivery. Arch Dis Child Fetal Neonatal Ed 2006; 91: 398-402.

107. Chadha NK, Zhang L, Mendoza-Sassi RA, César JA. Using nasal steroids to treat nasal obstruction caused by adenoid hypertrophy: does it work? Otolaryngol Head Neck Surg 2009; 140: 139-147.

108. Goldbart AD, Greenberg-Dotan S, Tal A. Montelukast for children with obstructive sleep apnea: a double-blind, placebo-controlled study. Pediatrics 2012; 130: 575-580.

109. Glockler-Lauf SD, Finkelstein Y, Zhu J, Feldman LY, To T. Montelukast and neuropsychiatric events in children with asthma: A nested case-control study. J Pediatr 2019; 209: 176-182

110. Kuhle S, Hoffmann DU, Mitra S, Urschitz MS .Antiinflammatory medications for obstructive sleep apnoea in children. Cochrane Database Syst Rev 2020; 1: CD007074.

111. Kheirandish L, Goldbart AD, Gozal D. Intranasal steroids and oral leukotriene modifier therapy in residual sleep-disordered breathing after tonsillectomy and adenoidectomy in children. Pediatrics 2006; 117: 61-66.

112. Don DM, Goldstein NA, Crockett DM, Ward SD. Antimicrobial therapy for children with adenotonsillar hypertrophy and obstructive sleep apnea: a prospective randomized trial comparing azithromycin vs placebo. Otolaryngol Head Neck Surg 2005; 133: 562-568.

113. Pirelli P, Saponara M, Guilleminault C Rapid maxillary expansion in children with obstructive sleep apnea syndrome. Sleep 2004; 27: 761-766.

114. Huang YS, Guilleminault C. Pediatric obstructive sleep apnea: Where do we stand? Adv Otorbinolaryngol 2017; 80: 136-144.

115. Camacho M, Noller MW, Zaghi S, Reckley LK, FernandezSalvador C, Ho E, et al. Tongue surgeries for pediatric obstructive sleep apnea: a systematic review and meta-analysis. Eur Arch Otorhinolaryngol 2017; 274: 2981-2990. 
Appendix 1 - Pediatric sleep questionnaire.

\begin{tabular}{|c|c|c|c|c|}
\hline \multicolumn{2}{|c|}{ While sleeping, does your child ... } & \multirow[t]{2}{*}{ Yes } & \multirow[t]{2}{*}{ No } & \multirow[t]{2}{*}{ DK } \\
\hline A1 & ... snore more than half the time/ & & & \\
\hline A2 & ... always snore? & & & \\
\hline A3 & ... snore loudly? & & & \\
\hline A4 & ... have "heavy" or loud breathing? & & & \\
\hline A5 & ... have trouble breathing, or struggle to breath? & & & \\
\hline \multicolumn{2}{|c|}{ Have you ever..... } & & & \\
\hline A6 & ... seen your child stop breathing during the night? & & & \\
\hline \multicolumn{2}{|c|}{ Does your child..... } & & & \\
\hline A7 & ... tend to breath through the mouth during the day? & & & \\
\hline A8 & $\ldots$ have a dry mouth during the day? & & & \\
\hline A9 & ... occasionally wet the bed? & & & \\
\hline \multicolumn{2}{|c|}{ Does your child ... } & & & \\
\hline A10 & ... wake up feeling unrefreshed in the morning? & & & \\
\hline A11 & $\ldots$ have problem with sleepiness during the day? & & & \\
\hline A12 & $\ldots$ has a teacher or other supervisor commented that your child appears sleepy during the day? & & & \\
\hline A13 & $\ldots$ is it hard to wake up your child in the morning? & & & \\
\hline A14 & ... wake up with headaches in the morning? & & & \\
\hline A15 & ... did your child stop growing on a normal rate at any time since birth? & & & \\
\hline A16 & $\ldots$ is your child overweight? & & & \\
\hline \multicolumn{2}{|c|}{ This child often ... } & & & \\
\hline A17 & $\ldots$ does not seem to listen when spoken to directly & & & \\
\hline A18 & $\ldots$ has difficulty organizing tasks or activates & & & \\
\hline A19 & $\ldots$ is easily distracted by extraneous stimuli & & & \\
\hline $\mathrm{A} 20$ & ... fidgets with hand or feet or squirms in seat & & & \\
\hline $\mathrm{A} 21$ & $\ldots$ is on the go 'or often act like if 'driven by a motor' & & & \\
\hline $\mathrm{A} 22$ & ... interrupts or intrude on others (namely, butts into others in conversations or games) & & & \\
\hline \multicolumn{5}{|c|}{$\begin{array}{l}\text { DK: do not know (scored 0), yes: symptoms present (scored 1). Copyright permission from Sleep Medicine. Chervin RD, Hedger } \\
\text { K, Dillon JE, Pituch KJ. Pediatric sleep questionnaire (PSQ): validity and reliability of scales for sleep-disordered breathing, snoring, } \\
\text { sleepiness, and behavioral problems. Sleep Med 2000; } 1: 21-31 . .^{50}\end{array}$} \\
\hline
\end{tabular}

